\title{
PROCESS AND SYSTEMS The rapid development and deployment of a new multidisciplinary CPAP service outside of a critical care environment during the early stages of the COVID-19 pandemic
}

\author{
Authors: Ryan E Robinson, ${ }^{\mathrm{A}}$ Rebecca Nightingale, ${ }^{\mathrm{B}}$ Freddy Frost, ${ }^{\mathrm{C}}$ Tom Green, ${ }^{\mathrm{D}}$ Gareth Jones, ${ }^{\mathrm{E}}$ Nneka Nwosu, ${ }^{\mathrm{E}}$ \\ Peter Hampshire, ${ }^{\mathrm{F}}$ Deborah Brown, ${ }^{\mathrm{G}}$ Michael Beadsworth, ${ }^{\mathrm{H}}$ Stephen Aston, ${ }^{\mathrm{H}}$ Angela Gillespie, ${ }^{\mathrm{I}}$ Mark Clark, ${ }^{ }$ \\ Tom Fletcher, ${ }^{\mathrm{H}}$ Neil Haslam, ${ }^{\mathrm{K}}$ Hassan Burhan ${ }^{\mathrm{E}}$ and Manish Gautam ${ }^{\mathrm{E}}$
}

The COVID-19 pandemic has led to a dramatic increase in patients presenting with type 1 respiratory failure. In order to protect our limited critical care capacity, we rapidly developed a new ward-based inpatient continuous positive airway pressure (CPAP) service with direct input from the respiratory, infectious diseases and critical care teams. Close collaboration between these specialties and new innovative solutions were required to facilitate this. CPAP equipment (normally reserved for domiciliary care) was adapted to reduce the pressure on our strained oxygen infrastructure. Side rooms on the infectious diseases ward were swiftly converted into new negative pressure areas using temporary installed ventilatory equipment, reducing the viral aerosol risk for staff. Novel patient monitoring solutions were used to protect staff while also ensuring patient safety. Staff training and specialist oversight was organised within days. The resulting service was successful, with over half (17/26 (65\%)) of patients avoiding invasive ventilation.

Authors: ${ }^{\text {A }}$ respiratory specialty trainee year 4, Royal Liverpool Hospital, Liverpool, UK and Liverpool School of Tropical Medicine, Liverpool, UK; ${ }^{\text {B }}$ respiratory physiotherapist, Royal Liverpool Hospital, Liverpool, UK and Liverpool School of Tropical Medicine, Liverpool, UK; C respiratory specialty trainee year 3, Royal Liverpool Hospital,

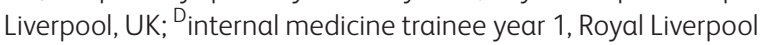
Hospital, Liverpool, UK; ${ }^{E}$ consultant in respiratory medicine, Royal Liverpool Hospital, Liverpool, UK; ${ }^{\text {F }}$ consultant in intensive care medicine, Royal Liverpool Hospital, Liverpool, UK; ${ }^{G}$ respiratory specialist nurse, Royal Liverpool Hospital, Liverpool, UK; ${ }^{{ }^{\prime}}$ consultant in infectious diseases, Royal Liverpool Hospital, Liverpool, UK and Liverpool School of Tropical Medicine, Liverpool, UK; ${ }^{\mathrm{I}}$ matron for infectious diseases and respiratory medicine, Royal Liverpool Hospital, Liverpool, UK; ' deputy general manager for respiratory medicine, Royal Liverpool Hospital, Liverpool, UK; ${ }^{\mathrm{K}}$ consultant in gastroenterology, Royal Liverpool Hospital, Liverpool, UK
KEYWORDS: COVID-19, CPAP, respiratory failure

DOI: 10.7861/fhj.2020-0167

The challenges posed by COVID-19

COVID-19 poses an unprecedented challenge to healthcare and has necessitated innovative approaches to managing the surge in demand for specific services. ${ }^{1}$ This has been felt most acutely in critical care units where it was predicted $20-30 \%$ of COVID-19 patients admitted to hospital would require require invasive mechanical ventilation due to respiratory failure. ${ }^{2}$ A collaborative decision was made by the respiratory, infectious diseases and the critical care teams to develop a new ward-based continuous positive airway pressure (CPAP) service in an attempt to prevent the critical care services from being rapidly overwhelmed. Similar approaches have been undertaken globally as the pandemic has developed and we have been fortunate to learn from other centres' experiences. ${ }^{3,4}$ The Royal Liverpool Hospital site $(\mathrm{RLH})$, one of two large inner-city secondary care sites that form Liverpool University Hospitals NHS Foundation Trust (LUHFT), has a total capacity of 650 beds with 36 critical care beds, of which, 18 offer invasive ventilation. Prior to this, the hospital's capacity to provide ward-based CPAP was limited to a critical care environment and one other specialised area (coronary care unit (CCU)).

There were significant barriers to facilitating this service in a timely manner during the evolving pandemic: the development and deployment of an appropriate area and equipment; the safe monitoring of patients receiving non-invasive ventilation (NIV); and mitigating the predicted increased risk of infection to staff. We describe our experiences of developing a new inpatient CPAP unit within days during the first stages of the evolving COVID-19 pandemic.

\section{Potential solutions}

Rapidly developing and deploying an inpatient CPAP suitable environment

The use of CPAP risks aerosolising SARS-COV-2 and is considered an aerosol generating procedure (AGP). ${ }^{5}$ The NHS and World 


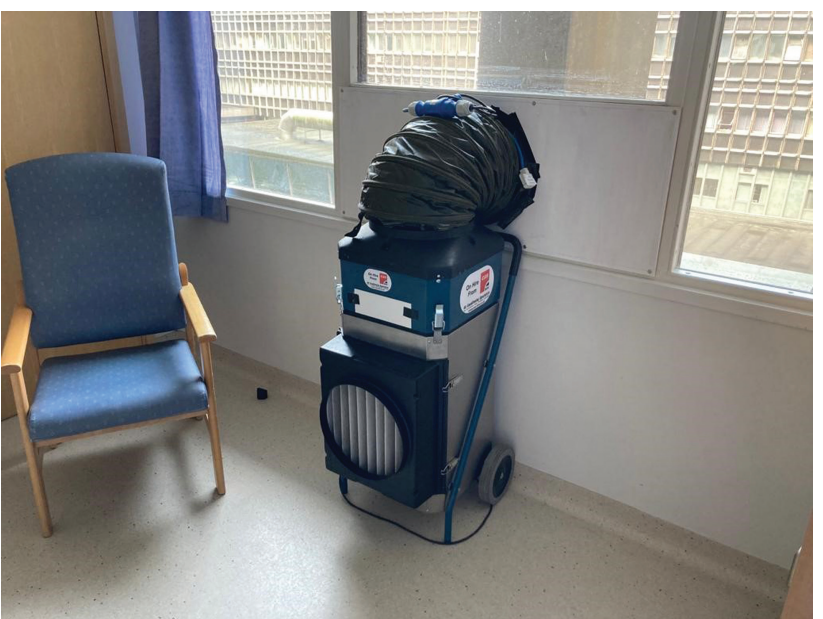

Fig 1. Temporary high-efficiency particulate absorbing filter in place to create a negative pressure environment.

Health Organization guidance recommends that AGPs are performed in a negative pressure room with air exchanges greater than 10 cycles per hour to minimise the risk of cross-infection to staff. ${ }^{6}$ The negative pressure side rooms on the isolation ward of the infectious diseases unit were identified as the best available area to perform ward based CPAP. The unit capacity was increased by six, to a total of 13 beds. This was achieved by converting the remaining side rooms into negative pressure areas through the installation of temporary industrial high-efficiency particulate absorbing (HEPA) filtered air purifying units, an innovative approach initially developed in the South Korean COVID-19 outbreak. This equipment allows air to ventilate externally, creating the required 10-15 air changes per hour required for a clinical negative pressure zone. Fig 1 displays the temporary filter system in situ.

Standardised non-vented masks covering the patient's nose and mouth were applied to ensure the staff were familiar with the new equipment. CPAP masks are typically vented so HEPA viral filters were fitted to the expiratory port of the circuit to reduce the viral aerosol risk as per the NHS guidance. ${ }^{6}$ A second viral filter was fitted directly onto the CPAP device. CPAP was initiated as per a dedicated standard operating procedure that has been described elsewhere. ${ }^{7}$ Dual oxygen ports were utilised as a rescue or bridge therapy when required to temporarily enable entrained oxygen flow rates beyond $15 \mathrm{~L} /$ minute.

\section{Avoiding system-wide oxygen pressure drop}

Another important consideration is the surging oxygen requirements for patients with COVID-19 and the strain that this can put on hospital oxygen systems and pipework. ${ }^{8}$ This is known to have almost occurred in at least one large UK hospital, temporarily closing the hospital to new admissions. ${ }^{9}$ CPAP using a pressure valve (such as the UCL-Ventura CPAP device) typically use oxygen as a driving gas to power the mechanism as well as to directly oxygenate the patient. A CPAP unit using this technology at full capacity risks rapidly overloading the hospital oxygen supply system, particularly if oxygen use elsewhere in the hospital is increased.

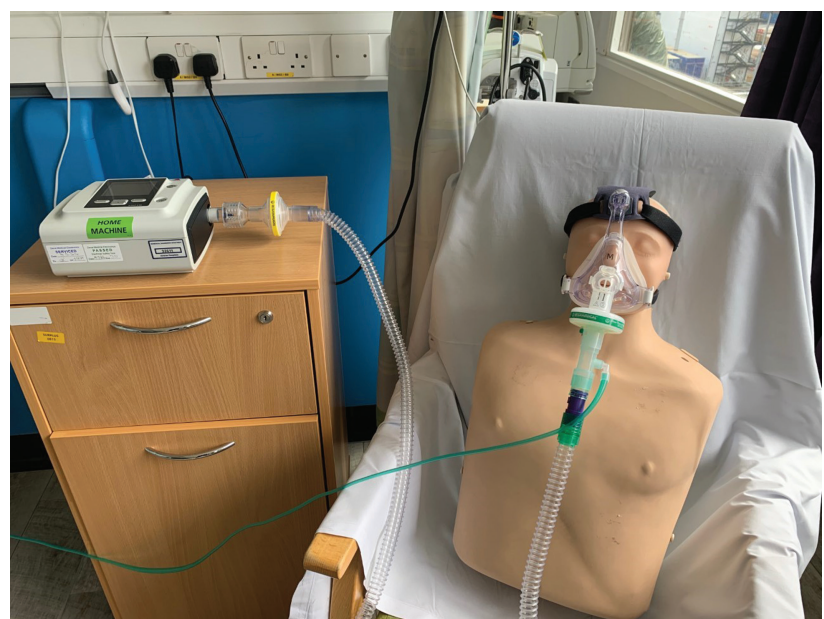

Fig 2. Domiciliary continuous positive airway pressure machine fitted with high-efficiency particulate absorbing filters.

To minimise the demand on the hospital oxygen infrastructure, a standard electrically powered non-invasive ventilator (Philips A30), a device which is often reserved for domiciliary CPAP, was utilised with wall oxygen entrained into the circuit as per the Association for Respiratory Technology and Physiology (ARTP) guidance (Fig 2). ${ }^{10}$ The oxygen consumption of this ventilator is $30-40 \mathrm{~L} /$ minute, which is $50-60 \%$ lower than the oxygen consumption of pressure valve-based machines used to deliver CPAP in the hospital. This equipment is readily available in many NHS trusts and, while it has less functionality when compared with ventilators used in a critical care setting, the relative simplicity of the machine made training staff easier.

\section{New methods of patient monitoring}

The Faculty of Intensive Care Medicine recommends a staffing ratio of 2:1 for patients requiring high-dependency unit level care, with similar levels recommended for NIV by the British Thoracic Society. ${ }^{11,12}$ Furthermore, the use of side rooms necessitated frequent donning and doffing of appropriate personal protective equipment (PPE). With staffing levels under pressure throughout the trust, and with predicted staff absence of up to $20 \%$ due to illness or self-isolation, maintaining this level of staffing ratio with appropriately trained individuals was not deemed feasible or sustainable. $^{13}$

To mitigate for this, changes were made to reduce staffing demands. Remote video monitoring relaying patients' vital signs to a central nursing station was installed, facilitating the observation of patients outside their individual negative pressure rooms. Patients provided verbal consent to real-time video monitoring on admission and its use was checked by the information governance team prior to installation to ensure it complied with trust guidance. No images were stored, and the real-time image could be turned off when required. Non-invasive monitoring was possible as the predominant issue was respiratory failure and vasopressors were not required. Video monitoring had the added advantage of restricting unnecessary PPE use at a time when hospital stock levels were precarious. 


\section{Ensuring staffing levels and providing sufficient training}

The ward was staffed by a team consisting of both respiratory and infectious diseases healthcare workers with direct input from a multidisciplinary team with experience in ventilation. To protect staff from COVID-19 AGPs, level 2 PPE was used, as per the latest Public Health England (PHE) guidance, and they were required to complete competencies in donning and doffing PPE before being involved in patient care. ${ }^{14}$ Specific training and attenuated NIV competencies, based on the standardised equipment and using trainers attending ward areas, were taken to avoid further exacerbating staff shortages. Both the respiratory and infectious diseases teams performed a combined twice daily ward round with extra consultant cover out of hours. Patients were assessed by the critical care team prior to transfer to the CPAP ward, enabling the rapid escalation of patients for invasive mechanical ventilation where deemed appropriate.

\section{Results so far}

Since the development of this service, 31 patients have received ward-based CPAP, of which, five patients were deemed not for intubation at the onset of CPAP. Over half (17/26 (65\%)) of patients avoided intubation through the use of CPAP; all have since been discharged. The median time on CPAP was 4 days (range 2-5). Eleven out of 26 (35.5\%) patients failed the trial of CPAP and required invasive mechanical ventilation in the intensive care unit. The median time to intubation in this group was 6 hours. Of the five patients deemed unsuitable for intubation, 2/5 (40\%) were weaned off CPAP and have subsequently been discharged, and $3 / 5(60 \%)$ died. During this period, there were no serious adverse events related to the provision of this CPAP service. A full description and further analysis of outcomes for this cohort have been described elsewhere. ${ }^{7}$

The number of patients requiring acute CPAP due to COVID-19 remained manageable with the described adaptions throughout the pandemic and, while significant, was less than the initial local modelling predictions. At the peak on 7 April 2020, we had a total of 209 COVID-19 inpatients across the trust. In our early experience, if CPAP is going to be ineffective this will become clear within the first 6 hours after initiation and case selection is critical. Larger multicentre studies are now ongoing to further clarify the optimal ventilation strategy in COVID-19 with respiratory failure. ${ }^{15}$

\section{Potential future}

This new service was developed within a period of days to manage an unclear but possibly very large number of patients during the first wave of the 2020 COVID-19 pandemic. To enable this, we accepted staffing ratios that, outside the context of a global pandemic, would be considered less than ideal. The use of CPAP in the management of COVID-19 remains disputed, however, from our limited local data thus far it appears to reduce the number of patients who would have otherwise required invasive mechanical ventilation.

We have overcome significant clinical and logistical barriers which, in a pre-pandemic setting, would have appeared insurmountable. Box 1 shows our key learning points. As admissions with severe COVID-19 reduced locally, the need for this intervention decreased and the CPAP service returned to critical care areas. However, when case numbers increased dramatically
Box 1. Key learning points from continuous positive airway pressure service provision

CPAP can be used for type 1 respiratory failure due to COVID19 pneumonia on a suitably adapted medical ward with novel solutions to enable safe monitoring.

In a negative pressure area with appropriate equipment, CPAP can be administered in an environment that minimises risk to staff.

Side rooms can be rapidly converted to negative pressure zones for AGPs using temporally installed ventilation equipment.

The use of relatively simple ventilation devices normally reserved for domiciliary CPAP can help reduce the hospital oxygen demand when compared with pressure valve-based CPAP options.

Close collaboration between medical specialties with support from critical care can enable the rapid development of new services in an unconventional setting.

$\mathrm{AGP}=$ aerosol generating procedure; $\mathrm{CPAP}=$ continuous positive airway pressure.

in our locality during October 2020 this service was urgently re-established within a 24 -hour period. Sustainability in staffing has been prioritised with the development of a comprehensive CPAP training programme for new and existing staff, ensuring the essential skill set required remains available. Contingency plans have been developed to open larger temporary negative pressure areas if required. As the UK COVID-19 epidemic continues to evolve, this service will need to retain its flexibility to ensure it continues to be responsive to patient needs.

\section{References}

1 Willan J, King AJ, Jeffery K, Bienz N. Challenges for NHS hospitals during COVID-19 epidemic. BMJ 2020;368:m1117.

2 Centers for Disease Control and Prevention. Interim clinical guidance for management of patients with confirmed coronavirus disease (COVID-19). CDC, 2020. www.cdc.gov/coronavirus/2019ncov/hcp/clinical-guidance-management-patients.html [Accessed 05 July 2020].

3 Westafer L, Elia T, Medarametla V, Lagu T. A Transdisciplinary COVID-19 early respiratory intervention protocol: an implementation story. J Hosp Med 2020;15:372-4.

4 Duca A, Memaj I, Zanardi F et al. Severity of respiratory failure and outcome of patients needing a ventilatory support in the emergency department during Italian novel coronavirus SARS-CoV-2 outbreak: preliminary data on the role of helmet CPAP and noninvasive ventilation. EClinicalMedicine 2020;24:100419.

5 Alhazzani W, Møller M, Arabi Y et al. Surviving Sepsis Campaign: Guidelines on the management of critically ill adults with coronavirus disease 2019 (COVID-19). Intensive Care Med 2020;46:854-87.

6 NHS England, NHS Improvement. Guidance for the role and use of non-invasive respiratory support in adult patients with COVID-19 (confirmed or suspected). NHS, 2020. https://amhp.org.uk/app/ uploads/2020/03/Guidance-Respiratory-Support.pdf [Accessed 05 July 2020].

7 Nightingale R, Nwosu N, Kutubudin F et al. Is continuous positive airway pressure (CPAP) a new standard of care for type 1 respiratory failure in COVID-19 patients? A retrospective observational study of a dedicated COVID-19 CPAP service. BMJ Open Respir Res 2020;7:e000639. 
8 World Health Organization. Oxygen sources and distribution for COVID-19 treatment centres: interim guidance. WHO, 2020. www. who.int/publications/i/item/oxygen-sources-and-distribution-forcovid-19-treatment-centres [Accessed 05 July 2020].

9 Norton T. UPDATED: Trust tells public to stay away after problems with oxygen equipment. Health Service Journal 2020. www.hsj. co.uk/coronavirus/updated-trust-tells-public-to-stay-away-afterproblems-with-oxygen-equipment/7027320.article [Accessed 23 June 2020].

10 Association for Respiratory Technology \& Physiology COVID Group. ARTP guidance for oxygen utilisation. ARTP, 2020. www.artp.org. uk/write/MediaUploads/Standards/COVID19/ARTP_Guidance_for_ Oxygen_Utilisation_Vers_4.0.pdf [Accessed 01 July 2020].

11 Faculty of Intensive Care Medicine. Core standards for intensive care units. Intensive Care Society, 2013.

12 Davies M, Allen M, Bentley A et al. British Thoracic Society quality standards for acute non-invasive ventilation in adults. BMJ Open Respir Res 2018;5:e000283.
13 Department of Health and Social Care. Coronavirus action plan. GOV.UK, 2020. www.gov.uk/government/publications/coronavirusaction-plan [Accessed 05 July 2020].

14 Public Health England. COVID-19: infection prevention and control (IPC). GOV.UK, 2020. www.gov.uk/government/publications/wuhannovel-coronavirus-infection-prevention-and-control. [Accessed 14 May 2020].

15 Warwick Clinical Trials Unit. RECOVERY RS Respiratory Support: Respiratory Strategies in COVID-19; CPAP, High-flow and Standard Care. Warwick CTU, 2020. https://warwick.ac.uk/fac/sci/med/ research/ctu/trials/recovery-rs [Accessed 23 June 2020].

Address for correspondence: Dr Ryan Robinson, Royal Liverpool Hospital, Prescot Street, Liverpool L7 8XP, UK. Email: ryan.robinson@lstmed.ac.uk 